\title{
Rheumatoid arthritis activity and severity in relation to commonly used contraceptive methods in a cohort of Egyptian female patients
}

Background: Objective: To study the impact/effect of commonly used contraceptive methods on
rheumatoid arthritis (RA) disease activity, severity, and damage in a cohort of Egyptian female RA patients.

Methods: Two hundred female RA patients were enrolled and divided into two groups; non-contraception users (50 patients) and contraception users (150 patients). The latter group was further subdivided according to the method of contraceptive method used into: 89 intrauterine device (IUD)-users; 45 combined oral contraceptives (COC)-users; 16 injectables-users. All patients underwent thorough history taking with special emphasis on contraception history, clinical examination, and assessment by routine laboratory tests, rheumatoid factor (RF) and antibodies to citrullinated protein antigens (ACPA). Rheumatoid arthritis disease activity was assessed using Disease Activity Score 28 using CRP (DAS28/ (RP), while RA disease severity was assessed using Rheumatoid Arthritis Severity Scale (RASS). Bilateral hand $\mathrm{x}$-rays were done and interpreted by the Short Erosion Scale (SES).

Results: The percentage of ACPA seropositivity among IUD-users was $75.3 \%$; the highest among all studied subgroups. IUD-users showed significantly higher ACPA titers $(p=0.020)$, as well as longer disease duration $(p=0.021)$ compared to other methods-users. The RASS was significantly higher in injectables-users in comparison to other methods-users followed by the IUD-users ( $p=0.008)$. COC-users had the least RASS. However, there were no significant differences regarding DAS28/CRP or SES between different contraceptive methods-users. There was positive correlation between ACPA titers and RASS.

Conclusion: The increased ACPA positivity as well as higher ACPA titers in women who are currently IUD-users suggests a possible etiopathogenic role for IUD in onset and perpetuation of RA disease. Mechanisms by which IUD could increase RA-related autoimmunity risk were discussed. Whether cessation of IUD use in RA patients might improve their current state of disease need further study.

Keywords: rheumatoid arthritis - contraception - DAS28/CRP • rheumatoid arthritis severity scale $\cdot$ short erosion scale $\bullet$ anti-citrullinated peptide antibodies

\section{Introduction}

Rheumatoid arthritis (RA) is a chronic inflammatory autoimmune disease; Highrisk genetic background, in combination with epigenetic marks and environmental exposures, leads to a cascade of events inducing synovitis and consequent destructive arthritis, as well as affecting a variety of extra-articular organs. The effects of environmental factors in genetically predisposed individuals are now of great significance in the pathogenesis and development of RA [1]. Pathologically, RA is a constellation of synovial inflammation and hyperplasia, autoantibody production: rheumatoid factor (RF) and anti-citrullinated protein antibody (ACPA), cartilage and bone destruction, as well as systemic features: cardiovascular, pulmonary, psychological, among others [2]. Autoantibodies: rheumatoid factor (RF) and antibodies to citrullinated protein antigens (ACPA) have important diagnostic and prognostic features and have proven very useful in clinical management of RA [3].

RA is characterized by an immune response against post-translationally modified proteins, in particular citrullinated proteins. Recent studies have found that the ACPA response matures shortly before clinical disease manifests itself and is characterized by an increase in titre, isotypeswitching, antigen-recognition profile, and a change in the Fc- glycosylation pattern [4].
Hala Lotfy Fayed*, Hatem H Eleishi, Heba A Kamal \& Nouran I Mahmoud

Kasr Alainy School of Medicine, Cairo University, Egypt

*Author for correspondence:

halafayed@yahoo.com 


\section{Genetic aspects}

The association between ACPA and HLADR alleles has gained special interest as these autoantibodies are highly prevalent in patients with RA and are strongly associated to a specific set of HLA-DR alleles specially HLADRB $1^{*} 04: 01$, ${ }^{*}$ 04:04, and *01:01 [5]. Special emphasis to the association with the HLA-DRB1 locus: It has been confirmed in patients who are positive for rheumatoid factor (RF) or antibodies against citrullinated peptides (ACPA); alleles that contain common amino acid motif (QKRAA) in the HLA-DRB1 region i.e. the shared-epitope, confer particular susceptibility, suggesting that some predisposing $\mathrm{T}$-cell repertoire selection, antigen presentation, or alteration in peptide affinity has a role in promoting auto-reactive adaptive immune responses [6]. This major dichotomy in genetic risk based on ACPA expression provides a molecular taxonomy for the rheumatoid arthritis syndrome, and explains why patients with ACPA-positive disease have a less favorable prognosis than those with ACPAnegative disease, suggesting a clinical usefulness for such molecular subsets [2].

\section{Epigenetic modification}

Despite the identification of several candidate gene variants by genetic mapping in RA, it cannot be explained by genetic susceptibility alone. Other stably heritable phenotype resulting from inheritable potentially reversible changes in DNA and chromatin without alterations in the DNA sequence 'epigenetic processes' is responsible for regulating gene expression. A combination of processes like chromatin methylation and posttranslational modification of histones or other chromatin-associated proteins among others represent these epigenetic modifications [5] that regulate gene expression through activation or silencing of genes [7].

\section{Environmental factors}

Still RA development is not fully explainable by genetic factors and epigenetic mechanisms alone, thus many environmental components have been proposed to be critically involved in the induction of the disease in geneticallypredisposed individuals. Various environmental factors; smoking; bacterial (e.g. Proteus sp. and Escherichia coli) and viral (e.g. Epstein-Barr virus, Cytomegalovirus) infections and their products e.g. heat shock proteins; microbiota: Porphyromonas gingivalis ( $P$. gingivalis), responsible for chronic periodontal infection has recently been connected to systemic disorders including RA [8] and Lactobacillus salivarius among other gut microbiota that was over-represented in RA patients and was present in increased amounts in cases of very active RA [9]; as well as hormones can trigger citrullination both in articular and extra-articular locations which progresses to specific immunity and disease in geneticallypredisposed individuals leading to increased expression in peptidyl-arginine deiminase (PAD) enzyme with consequent generation of protein citrullin through various mechanisms including molecular mimicry, epitope spreading, or generation of new citrullinated epitopes not recognized by 'self ...etc. [10]; only two out of the five human PAD subtypes (PAD2 and PAD4) are relevant for RA, as they are the ones expressed in joints, immune cells, neutrophils and mast cells [11].

In citrullination or deimination, PAD enzymes catalyze protein transformation of arginine into peptidyl-citrulline substituting an imine nitrogen group on arginine residues with an oxygen group. As consequence of either reaction, the protein loses its positive charge, which affects the intramolecular bonds and leads to conformational changes that alter the protein function and make it more susceptible to proteolytic degradation. The most accused PAD isozyme in RA pathogenesis is peptidyl arginine deiminase, type 4 (PAD4) which regulates gene expression through histone modifications i.e. post-translational processing or post-translational modification that results in quantitative or qualitative alterations in citrullination of mucosal proteins. When PAD converts arginine to citrulline on a histone, it blocks further methylation of the histone, inhibiting transcription. An ACPA response detected with a diagnostic anti-cyclic citrullinated peptide assay represents loss of tolerance to these neoepitopes [2]. This PAD-mediated citrullination is not only a basic cellular damage, but also an inflammatory process occurring in a range of inflammatory backgrounds such as synovial tissue, gingival and periodontal sites and lungs, challenging local immunity. This explains why ACPA is not only considered as diagnostic biomarker for RA and a prognostic factor for severe aggressive disease, but also confer a pathogenic role [12]. Here it is worth mentioning that only individuals with definite HLA polymorphisms, such as the conserved region of HLA-DRB1 alleles are at risk to recognize such citrullinated proteins as "nonself" and to develop RA [13]. 
This dysregulated PAD4 citrullination, leads to perpetuation of inflammation and the generation of new citrullinated self-epitopes that could trigger the production of autoantibodies and mediate tissue destruction. Moreover, PAD4 is involved in formation of neutrophil extracellular traps (NETs) and may play a role both in generating potential auto-antigens i.e. the newlycitrullinated antigens and in host defense against bacterial infections [7].

Despite the fact that citrullination is normally involved in many physiological processes in a variety of tissues, dysfunctional posttranslational protein modifications are often reported during autoimmune pathology as in RA, leading to the loss of the immunological tolerance and stimulation of a specific profile of humoral response [14]. This abnormal citrullination of endogenous proteins represents an initial, tactical process in the RA complex pathobiology, that drives the induction of altered self-epitopes and extensive autoimmune response [15].

\section{Role of sex Hormones}

The greater risk of RA among women than men as well as its tendency to affect women more than men (the female to male ratio is around 3 to 1 ), suggests that sex hormones may have an influence on disease [16]. This has led to much interest into whether or not there is hormonal influence on disease risk.

This observation shows a link between the hypothalamic-pituitary-adrenal axis and cytokine production; the central nervous system is normally involved in immune regulation and homeostasis, as well as neuro-immunologic interactions that regulate disease development in autoimmune disease. These effects may operate locally or centrally [2].

In addition, sex hormones appear to modulate the immune/inflammatory responses by various mechanisms both in female and male patients with RA, with estrogens being enhancers at least of humoral immunity and androgens and progesterone being natural immune suppressors [17]. This is evidenced by the higher concentrations of estrogens and lower concentrations of androgens reported in synovial fluid from RA patients of both sexes which support the possible modulatory roles in synovial tissue hyperplasia and chronic synovial cell activation, resulting from the effects of estrogen on cell proliferation and apoptosis [18].
As the pro-inflammatory cytokines (TNF $\alpha$, IL1 , and IL-6) accelerate the metabolic conversion of estrogens from androgens leading to increase in estrogen concentrations in RA synovial fluid from patients of both sexes, locally-increased pro-inflammatory estrogen levels might exert activating effects on synovial cell proliferation, including macrophages and fibroblasts. Further reduction in the availability of anti-inflammatory androgens in local tissues may be due to stimulation or further activation of synovial cells to produce cytokines $[19,20]$.

Schmidt have demonstrated steroid hormone conversion in primary cells of synovial origin from RA patients suggesting a dual proinflammatory and anti-inflammatory effects of estrogens in human inflammation depending on numerous factors: the immune stimulus and subsequent antigen-specific immune responses, the cell types involved during different disease phases, the specific microenvironment, the timing of estrogen administration in relation to the disease course (plus the reproductive status in women), the estrogen concentration, the estrogen receptor expression variability depending on the microenvironment/cell type, and intracellular conversion of estrogens leading to important biologically active metabolites with quite different proinflammatory and antiinflammatory functions [21]. Intracellular conversion of estrogen to downstream metabolites in synovial cells from RA patients has been documented in various studies $[22,18]$.

Oral contraceptives (OCs) among various other factors including the menstrual cycle, pregnancy, the postpartum period, menopause, chronic stress, the use of corticosteroids, and steroid hormone replacement can change the sex hormone milieu and/or peripheral conversion, resulting in alteration of androgen to estrogen ratios and modulation of disease [23].

Hence, genetic-epigenetic-environment interactions promote loss of tolerance to self-proteins that contain a citrulline residue, resulting in anti-citrulline response that can be detected in T-cell and B-cell compartments and is probably initiated in bone marrow or secondary lymphoid tissues. Thereafter, localization of the inflammatory response takes place in the joints by mechanisms that mostly involve microvascular, neurologic, biomechanical, or other tissue-specific pathways i.e. synovitis is initiated and perpetuated by positive feedback loops and in turn promotes systemic disorders that make up the RA syndrome Figure 1 [2]. 


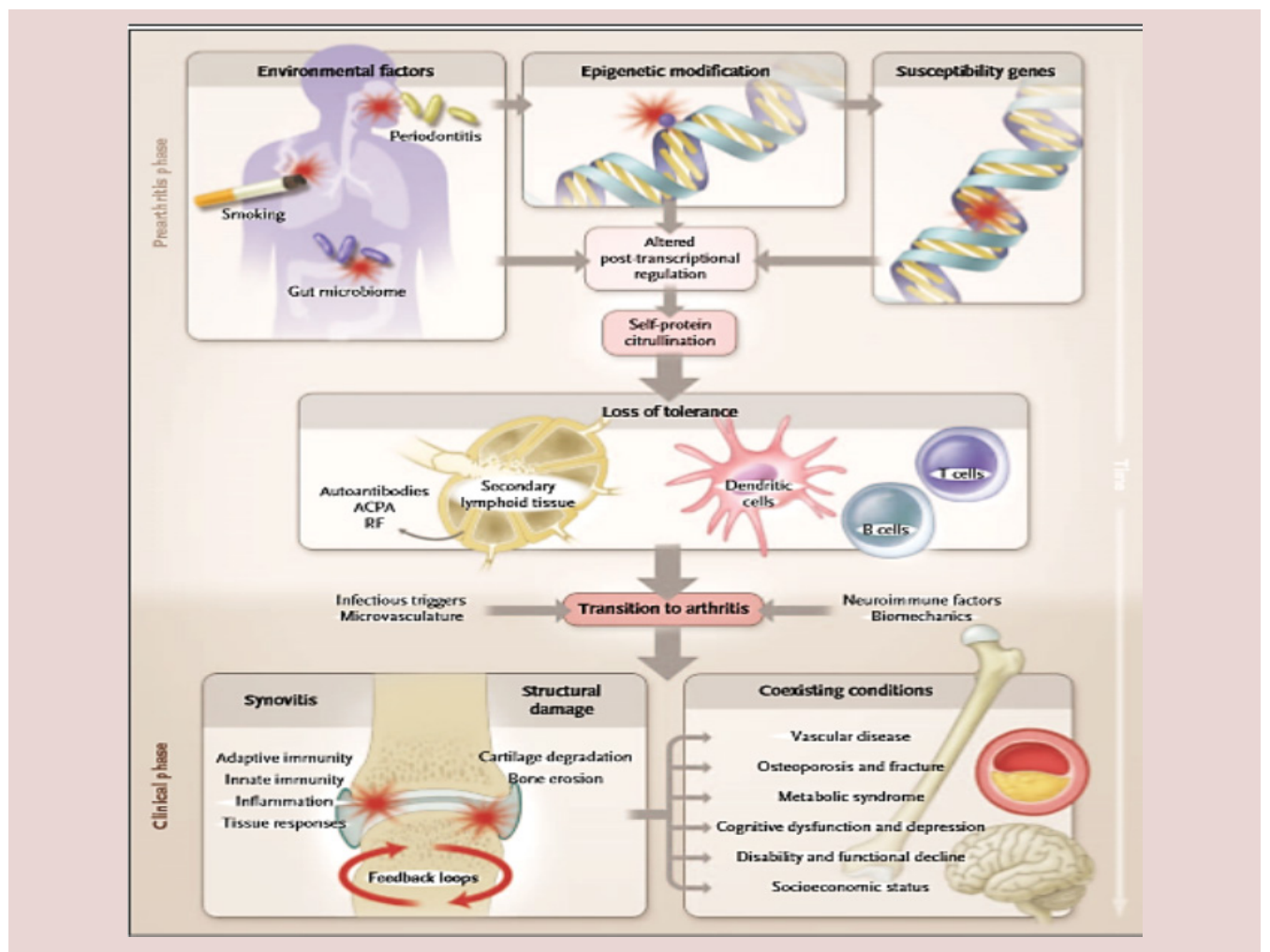

Figure 1. Multistep progression in development of RA (Mclnnes and Schett, 2011)

Innate immune response: NETosis and autophagy

The contribution of neutrophil extracellular trap (NET) formation (NETosis) to the breaking of immunological tolerance and the maintenance of autoimmunity and inflammation in RA has gained much interest in recent years. It consists of the release of intracellular components, including DNA, histones and an array of proteins that all together create a net-like structure outside the cell. NETs trap and restrain invading pathogens and use their highly localised focus of antimicrobial granular peptides to degrade virulent factors and even kill microorganisms [5].

It was found that neutrophils from RA peripheral blood (PB) and synovial fluid (SF) display an increased NETosis in basal conditions or after stimulation with serum antibodies or inflammatory cytokines together with nuclear translocation of PAD4 leading to PAD4mediated citrullination of $\mathrm{H} 3$, and altered nuclear morphology.

Many cytoplasmic and extracellular citrullinated antigens that represent targets of ACPA, and immune complex formation and that might act as inducers of further NETosis in RA have been described among NET components [24] and could represent a source of citrullinated antigens, fueling the ACPA autoimmune response within the RA synovium [25].

Moreover, autophagy which is a necessary process for the turnover of organelles, the maintenance of cellular homeostasis and the regulation of cell fate has been proposed as a cellular mechanism involved in the pathogenesis of RA, and contribution to its progression [26]. A role of autophagy in the generation of citrullinated peptides has been demonstrated [27,28].

Novel mechanisms involved in the citrullination of ACPA have been described in recent years as the ability of periodontal disease bacterium Porphyromonas gingivalis to induce citrullination via $\mathrm{PAD}$ production that is consequently relevant in the pathogenesis of RA [29]. The link between Porphyromonas gingivalis-derived ACPA and RA activity has also been suggested [30].

Furthermore, a positive correlation between serum antibody titre directed towards citrullinated-HSP60 (citHSP60) and bone damage scores, suggesting the active role of ACPA and osteoblast surface-expressed citHSP60 specifically implicated in the pathogenesis of RA [31]. 


\section{Contraceptive methods}

The issue of contraception and contraceptive method safety is of paramount importance in female patients with RA given the fact that the disease onset often occurs during the reproductive years. In addition, RA treatments like methotrexate, leflunomide, among others are teratogenic, necessitating highly effective contraception for sexually-active females [32].

The choice of proper contraceptive method is of paramount importance in female patients with rheumatoid disease, as these are most often the patients with the greatest need for effective contraception use. Knowledge of risks and benefits of contraceptive methods in the general population has improved, as has the safety and effectiveness of hormonal contraceptives. Contraception associated mild adverse effects are far outweighed by the significant risks associated with unintended pregnancy in women with active inflammatory disease on immunosuppressive medications.

\section{Oral contraceptives}

\section{Combined Hormonal Contraceptives}

Combined (estrogen-progesterone) hormonal contraceptives are currently available with different administration methods, but they share the same mechanism of action. There are the orally administrated forms, the transdermal patches and the intravaginal rings Combined oral contraceptives (COCs) differ in the amount of estrogen (ranging from 20-50 $\mu \mathrm{g}$ ), type of progesterone, and dose throughout the menstrual cycle: the most commonly used preparation is a combination of the synthetic estrogen is ethinylestradiol $(20-50 \mu \mathrm{g})$ and the progestin is one of the multiple $17-\alpha$ ethinyl analogs of 19-nortestosterone. Newer generation progestines were developed to decrease androgenic side effects and increase progestational effects [33].

Combined oral hormonal contraceptives have multiple effects on the reproduction system that account for the high rate of effectiveness with perfect use: ovulation is blocked due to inhibition of the mid-cycle surge of gonadotropins, sperm penetration is inhibited because of changes in cervical mucus, uterine and tubal motility is altered, and blastocyst survival is impaired by inhibition of endometrial gland function [34].

While purely theoretical, given the fact that hormone studies in RA patients do not suggest unusually low endogenous estrogen levels: rather, they show normal estrogen levels with low androgen levels: low levels of gonadal and adrenal androgens (testosterone and dehydroepiandrosterone) have been demonstrated in serum and synovial fluid of RA patients of both sexes [23], a case could be made for use of COCs containing the relatively more androgenic (second generation or higher) progestins, to maximize androgenic immunosuppression, if one is hoping for a noncontraceptive disease modifying benefit. In terms of the particular method chosen for RA patients, combined oral hormonal contraceptives or the transdermal patch may be effective and especially convenient for patients with RA [33].

There is some evidence that prior use of oral contraceptives is associated with a lower incidence of RA, whereas other reports do not support such protective effect; the results of many controlled studies have been contradictory, and there was no consensus for many years regarding the relationship of COCs to the prevention of RA or exacerbation of the rheumatoid disease [33]. However, recently, considerable interest has focused on whether oral COCs are protective against the risk of developing RA: the most recent study of Swedish Epidemiological Investigation of Rheumatoid Arthritis (EIRA), 2017 which included women aged 18 and above, living in a defined area of Sweden between 1996 and 2014 revealed that the ever and past COC use decreased the risk of RA, especially ACPA-positive RA. Besides, a long duration of COC use decreased the risk of both disease subsets, suggesting that combined hormonal contraceptive methods represent a safe and effective option for patients with RA [35].

Intrauterine device (IUD)

The intrauterine device (IUD) is the most commonly used form of reversible contraception worldwide; available in a non-medicated (inert) form the copper-containing IUD (Cu-IUD/ Copper T380) or medicated form IUD with a levonorgestrel-releasing intrauterine system (LNG-IUD) [35]. Being placed by a physician for 5-10 years, it offers convenient and long-lasting protection, however, they are considered as a foreign body inside the uterine cavity producing varying degrees of foreign body reactions that produce signs of a low grade inflammatory response, including leucocyte infiltration in the endometrium. 
There are some possible mechanisms of action of $\mathrm{Cu}$-IUD including induction of inflammatory foreign-body response in the endometrium that whose cellular and humoral components are released into the uterine cavity, creating a toxic intrauterine milieu that affects the function or viability of gametes, decreasing the rate of fertilization and lowering the chances of survival of any embryo that may be formed, even before it reaches the uterus, together with the spermicidal action of the copper ions released from [36], in addition to local changes due to release of medication in case of LNG-IUD that causes thickening in consistency of cervical mucus, altering sperm migration. LNG-IUDs also result in endometrial suppression [37].

The suggested mechanisms of the cellular and biochemical responses to the intrauterine foreign body placed in the sterile environment of the endometrial lumen involve two reactions; Short-term inflammatory response: a transient low-grade bacterial infection of the endometrial cavity for one to two days immediately following insertion of an IUFB. This infection results from the transfer of certain bacteria normally found in the cervical mucus to the endometrial lumen; the most common bacterial species in cervical mucus are Staphylococci, Streptococci and Diphtheroids. Even though these bacteria are rapidly destroyed, their presence increases the number of inflammatory cells in the uterine cavity. The synergistic chemotactic effects of both live and dead bacteria together with the IUFB reaction produce a more rapid influx of intrauterine inflammatory cells than would normally occur in the absence of an IUFB; Longterm inflammatory response: occurs due to the presence of the IUD long enough to induce morphological, ultrastructural, physiological and biochemical changes in the epithelial and subepithelial layers. This cyclic FB reaction is continually renewed due to cyclic decidualization of the endometrial surface resulting in cyclic chronic endometritis produced by the IUD: the sloughing of tissue and the biochemical events associated with menstruation do not in themselves eliminate these chronic inflammatory cells; the stratum basalis of the endometrium as well as focal areas of superficial endometrium may remain during menstruation, so it is likely that foci of chronic inflammatory cells may remain in the endometrial tissue which does not slough at menstruation. In an effort to expel the IUD, the myometrium exerts a contractile force, and the foreign body is displaced within the uterine cavity; the mechanical movement of the foreign body over the surface of the endometrium is partially responsible for some of the associated histological alterations. The formation of a small bleb on the surface of the endometrium with fluid formation elevating the superficial epithelium appears similar to formation of a cutaneous blister. As the bleb ruptures and expels the fluid, the superficial epithelium is denuded with formation of an ulcerated area, exposing the superficial stroma. The superficial erosions are detected by scanning electron microscopy. As a result, a series of morphological and physiological changes occur in the endometrial tissues and cells in response to the insertion of an IUD ending in abnormal inflammatory infiltration of the endometrium that under normal conditions, harbors no inflammatory cells, except for a few scattered mononuclear cells in the stroma at the late luteal and menstrual phase of the cycle [38].

On the basis of a systematic review of studies published between 2002 and 2015 concerned with the microorganisms that can colonize the IUD, Martins and colleagues in 2016 found an association between the use of IUD and some genital infections such as bacterial vaginosis. The most frequent pathogens observed in the method's users are Actinomyces ssp., Prevotella spp., and Mycoplasma hominis; however, the main acute pelvic inflammatory disease occasioner is Actinomyces spp (14 times greater risk for the presence of Actinomyces spp. in IUD users than in non-users), creating an environment of microbiome imbalance which might reach the upper genital tract. The prolonged use of IUD can also cause the imbalance in the vaginal bacterial flora, enabling the proliferation of anaerobic microorganisms as Gardnerella vaginalis, predisposing to the emergence of bacterial vaginosis [39]. Madden and coworkers earlier in 2012 also found that IUD use, was significantly associated with bacterial vaginosis $(\mathrm{BV})$ that can ascend to alter uterine microbiome [40]. A cross-sectional study in a population of 3870 smears of women who attended three institutions providing health services showed a high prevalence of vaginal actinomycosis in intrauterine device users followed by coinfections with bacterial vaginosis, candidiasis or trichomoniasis [41]. Kim and colleagues in 2014 showed association between pelvic actinomycosis with IUDs and ascending infection from the uterus in cervical smears. Although it is present in the normal flora of the mouth, the pharynx and probably the lower 
ileum and cecum, however, if the integrity of the mucosal membrane is broken, human infection may occur and can cause a fatal disease in some cases [42].

Additionally, the association of an increased risk of ACPA positivity with IUD use in first degree female relatives of known RA patients was suggested in the Studies of the Etiology of Rheumatoid Arthritis (SERA): elevated levels of anti-CCP was found in the blood of first degree female relatives of known RA patients using IUD several years before joint symptoms develop in RA, which strongly predicts future development of a joint disease and a diagnosis of RA; this period of RA development is often called the preclinical period of RA. This ACPA positivity is theoretically attributed to the IUD-induced endometrial inflammation that may be a potential mucosal trigger of RA-related antibodies, and recent data suggest that ACPA may be directly pathogenic. Therefore, it is important to understand factors that influence the development of these RArelated autoantibodies, even in the absence of inflammatory arthritis [43].

\section{Injectable Depomedroxyprogesterone Acetate (DMPA)}

The progesterone-only contraceptive depomedroxy-progesterone acetate (DMPA) is a suspension of microcrystals of a synthetic progestin that acts by the inhibition of ovulation with the suppression of FSH and LH levels and eliminates the LH surge. This results in a relative hypoestrogenic state. It is administered as an intramuscular injection every 12-14 weeks, making it a convenient option that requires little effort on behalf of the patient in relation to administration and adherence, and has superior efficacy to progestin-only oral contraceptives. A subcutaneous version of the drug is also available, that delivers a lower dose of medroxyprogesterone acetate (MPA) than does the intramuscular formulation (104 mg vs. $150 \mathrm{mg}$ ). The most important concern for use of DMPA especially with RA patients with higher risk for osteopenia (both from disease systemic inflammation, as well as from medications namely glucocorticoids) is the occurrence of reversible bone loss due to inhibition of ovulation: the reduction in bone density in healthy women is $5.7-7.5 \%$ after 2 years of use according to the American College of Obstetricians and Gynecologists Committee on Gynecologic Practice in 2008. Therefore, it would not seem a good long-term choice for the RA patient especially those receiving long-term corticosteroid therapy with a history of, or with risk factors for, non-traumatic fractures [33].

A cross-sectional study was undertaken in 2474 women aged 30-34 years from Bangladesh, Brazil, China, Egypt, Mexico, Thailand and Zimbabwe to evaluate the effect of the use of hormonal contraceptives on bone mass, and showed that among current users of combined oral contraceptives, bone mineral density (BMD) was significantly higher for short-term users (two-three years) as compared with never-users. Among short-term users of the progestogenonly methods (DMPA and Norplant), BMD was significantly lower compared with neverusers, suggesting that the use of hormonal contraceptives by young adult women is associated with small changes in BMD that occur early after initiation of use and are reversible. In view of these findings and other data indicating that progestogen-only contraceptives have a small but unfavourable effect on bone mass [44].

\section{Hypothesis and aim of work}

We hypothesized that the IUD might be a potent environmental trigger through its potential to induce chronic subtle renewed tissue injury and chronic inflammation triggering citrullination at the site of the endometrium thus aggravating and perpetuating RA disease in female RA patients.

The aim of this work was to study the impact/ effect of commonly used contraceptive methods on rheumatoid arthritis (RA) disease activity, severity, and damage in a cohort of Egyptian female RA patients.

The study tried to explore the association of the RA disease activity (and/or) severity and the commonly used female contraceptive methods in a cohort of Egyptian female patients, trying to provide better insights in choices of effective contraception among sexually active RA patients.

\section{Patients and methods}

In this Cross-sectional study, we enrolled 200 female patients with rheumatoid arthritis who were recruited from the Rheumatology and Rehabilitation outpatient clinic at Kasr Al Ainy Hospital.

\section{Inclusion criteria}

- Female patients diagnosed as rheumatoid arthritis and fulfilling the American College of Rheumatology (ACR) and the European League Against Rheumatism (EULAR) classification criteria 2010 [45]. 
- Age $\geq 18$ years old

- Being of at least 2 years after menarche

- For contraceptive method users: use of specific method for contraception for at least 6 months

- For non-contraceptive method users: being on no contraception method for at least 6 months

\section{Exclusion criteria}

- Age $<18$ years old

- Onset of menarche of less than 2 years

- Use of specific contraceptive method for less than 6 months

- Irregular use of contraception method within the studied 6 months

- Severe liver or kidney dysfunction

- Any sort of malignancies or treatment with chemotherapy, hormonal therapy and radiotherapy

- Hyperprolactinemia

- Patients with morbid obesity (BMI >40)

- Pregnancy or lactation

- Menopause

All the eligible female patients received a consent form outlining their right to refuse to participate in or withdraw at any time from the study and have signed for their acceptance of being enrolled in the study.

The 200 enrolled patients were divided into two groups; non-contraception users (50 patient) and contraception users (150 patients). The latter group was further subdivided into IUD users, COC users and injectables users.

History taking including menstrual and contraceptive history: age at menarche, regularity of menses, and history and state of contraceptive methods use (previous/ current); IUD, COC, injectables or non-user, and clinico-laboratory evaluation; assessment of RA disease activity using Disease Activity Score 28 based on C-reactive protein (DAS28/CRP) [46], as well as, disease severity using Rheumatoid Arthritis Severity Scale (RASS): a brief, physician-completed scale consisting of three visual analogue scales using $10 \mathrm{~cm}$ lines representing disease activity, functional impairment and physical damage [47] were done.
Patients were subjected to the following laboratory investigations: Complete blood count [48], Erythrocyte sedimentation rate (ESR) done by Westergren technique, taking the reading of the first hour [49], C-reactive protein (CRP): measured in $\mathrm{mg} / \mathrm{L}$, with a reference for acute phase response up to 6 $\mathrm{mg} / \mathrm{L}$ [48], liver function tests: serum alanine transaminases (ALT), aspartate transaminase (AST) [48], serum creatinine level: normal mg/ $\mathrm{dL}$ [48], rheumatoid factor (RF): using a latexenhanced nephelometric assay with human and rabbit IgG-coated latex beads as antigen [50], antibodies to cyclic citrullinated peptides (ACPA): using enzyme linked immunosorbant assay (ELISA) [51].

\section{Radiographic scoring}

Radiographs of the hands and wrists (posteroanterior view) of all participating individuals were obtained at the time of study entry, unless films obtained within one year prior to entry were available for review. All radiographs were read by a single radiologist who was blinded to patients' clinical and genetic information, to document the presence and extent of erosive disease and then calculate the Short Erosion Scale (SES) [52].

\section{Statistical analysis}

Data were statistically described in terms of mean \pm standard deviation $( \pm S D)$, median and range, or frequencies (number of cases) and percentages when appropriate. Comparison of numerical variables between the study groups was done using Student t-test for independent samples in comparing 2 groups, and one way analysis of variance (ANOVA) test with post-hoc multiple 2 group comparisons when comparing more than 2 groups. For comparing categorical data, Chi-square $\left(\chi^{2}\right)$ test was performed. Exact test was used instead when the expected frequency is less than 5 . Correlation between various variables was done using Pearson moment correlation equation for linear relation in normally distributed variables and Spearman rank correlation equation for non-normal variables/ non-linear monotonic relation. "p" values less than 0.05 was considered statistically significant. All statistical calculations were done using computer program IBM SPSS (Statistical Package for the Social Science; IBM Corp, Armonk, NY, USA) release 22 for Microsoft Windows. 


\section{Results}

Two hundred RA female patients took part in this cohort study. They were divided into two groups; non-contraception users (50 patients), as a control group and contraception users (150 patients). The latter group was further subdivided into IUD users, oral contraceptives users and injectable users.

\section{Non-contraception users}

This group comprised 50 RA female patients, who were on no contraceptive method for $>6$ months. Their age ranged from 19 to 54 years; mean age \pm SD $(39.16 \pm 9.73)$ years. Disease duration ranged from 0.5 to 22 years with a median of 3 years. Thirty patients $(60 \%)$ were RF seropositive and 35 patients (70\%) were ACPA seropositive.

\section{Contraception users}

This group comprised 150 RA female patients who were on a contraceptive method for $>6$ months. Their age ranged from 23 to 49 years; mean age $\pm S D(37.03 \pm 6.61)$ years. Disease duration ranged from 0.5 to 25 years with a median of 3 years; mean disease duration \pm SD $(4.76 \pm 4.74)$ years. They were 89 patients on IUD (59.33\%), 45 patients on COC (30\%), 16 patients on injectables $(10.67 \%)$. Eighty four patients (56\%) were RF seropositive and 99 patients (66\%) were ACPA seropositive.

Of all the studied patients, 145 (72.5\%) had regular menstrual cycles. The age of menarche ranged between 10 -18 years, with mean \pm SD $13.07 \pm 1.44$ years Table 1 .

Among the studied group, the duration range of IUD first use before the onset of RA 0.25-26 years, with median of 8 years. Also, 151(75.5\%) of all patients used IUD prior to the onset of RA Table 2.

The patients who used IUD someday prior to the onset of RA had statistically significant higher ACPA titers than those with disease onset preceding IUD use $(\mathrm{p}=0.006)$ Table 3.

There is a significant positive correlation between the duration passed since first exposure to IUD and the disease duration i.e. the earlier the first exposure to IUD, the longer the disease duration with $p$ value $<0.001$ and $r=0.328$, as shown in Figure 2 .

There is a statistically significant difference in disease duration in current IUD users compared to other subgroups of the study i.e. the current IUD users have the longest disease duration, with overall $\mathrm{p}=0.02$. Also, the disease duration in IUD users was significantly higher than COC users with $\mathrm{p}=0.04$ Table 4 .

Among all the users, $122(61 \%)$ were RF seropositive and $134(67 \%)$ were ACPA seropositive; the number (\%) of IUD users who were ACPA seropositive was 67/89(75.3\%), compared to $22 / 45(48.9 \%)$ among COC users, and 10/16 (62.5\%) among injectable user Table 5 .

\begin{tabular}{|c|c|c|c|c|}
\hline Table 1. Demographic characteristics of studied patients & & \\
$\begin{array}{c}\text { Variable } \\
\text { (Mean } \mathbf{\text { SD) }}\end{array}$ & Non-users (N=50) & IUD-users (N=89) & COC-users (N=45) & $\begin{array}{c}\text { Injectables users } \\
\text { (N=16) }\end{array}$ \\
\hline Age & $39.16 \pm 9.73$ & $37.39 \pm 7.06$ & $35.80 \pm 6.163$ & $38.44 \pm 5.06$ \\
\hline Age of menarche & $12.74 \pm 1.32$ & $12.78 \pm 1.27$ & $13.67 \pm 1.33$ & $13.93 \pm 1.98$ \\
\hline Disease Duration & $5.34 \pm 5.38$ & $5.51 \pm 5.46$ & $3.24 \pm 1.98$ & $3.03 \pm 1.64$ \\
\hline
\end{tabular}

\begin{tabular}{|c|c|c|c|c|}
\hline \multicolumn{2}{|c|}{ Table 2. Onset of RA in relation to prior IUD use } & \\
\hline $\begin{array}{c}\text { No. of patients (\%) } \\
\text { Non-users (N=50) }\end{array}$ & IUD-users (N=89) & COC-users (N=45) & $\begin{array}{c}\text { Injectables-users } \\
\text { (N=16) }\end{array}$ \\
\hline $\begin{array}{c}\text { IUD use someday before RA } \\
\text { onset }\end{array}$ & $25(50 \%)$ & $86(96.6 \%)$ & $28(62.2 \%)$ & $12(75 \%)$ \\
\hline RA onset before IUD use & $1(2 \%)$ & $3(3.4 \%)$ & $0(0 \%)$ & $0(0 \%)$ \\
\hline No prior IUD use & $24(48 \%)$ & $0(0 \%)$ & $17(37.8 \%)$ & $4(25 \%)$ \\
\hline
\end{tabular}

Table 3. Onset of RA in relation to prior IUD use
\begin{tabular}{|c|c|c|}
\hline Used IUD someday prior to RA onset? & RF titer (IU/mL) & ACPA titer (IU/mL) \\
\hline Yes (Mean \pm SD) & $63.31 \pm 79.84$ & $176.10 \pm 180.42$ \\
\hline No (Mean \pm SD) & $94.07 \pm 147.79$ & $108.80 \pm 132.74$ \\
\hline p value & 0.169 & $p=0.006^{*}$ \\
\hline & p value is significant $<0.05$ & \\
\hline
\end{tabular}


Research Article Fayed, Eleishi, Kamal, et al.

As regards titers for ACPA among various subgroups, it was found that ACPA titer was significantly higher in IUD users compared to other subgroups in the study, with overall $\mathrm{p}$ value $=0.021$ as shown in Table 6 . Furthermore, it was significantly higher among IUD users compared to COC users $(\mathrm{p}=0.04)$.
There was significant difference in RASS between the four subgroups with overall $\mathrm{p}$ value $=0.008$ Table 7. It was higher in the injectables users followed by the IUD users; The COC users had the least RASS. Moreover, the RASS in IUD users was also significantly higher than nonusers ( $p$ value $=0.02$ ). However, SES showed no

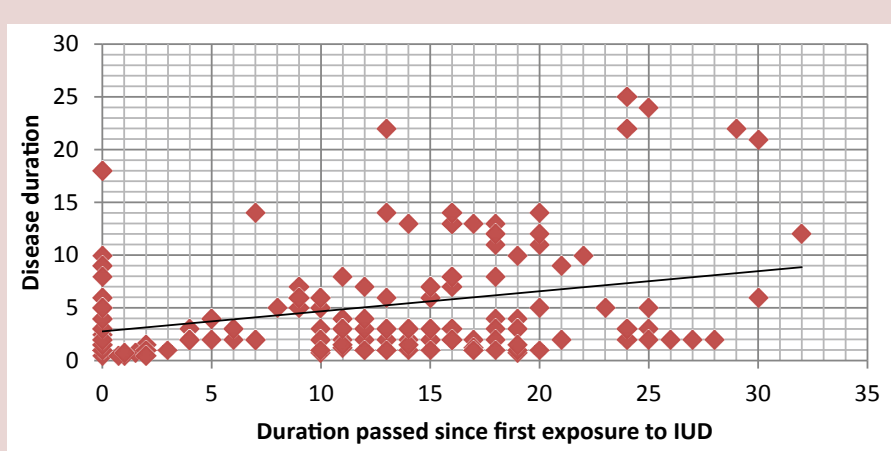

Figure 2. Correlation between the duration passed since first exposure to IUD and the disease duration

Table 4. Disease duration of studied subgroups
\begin{tabular}{|c|c|c|c|c|c|}
\hline Disease duration & Non- users (N=50) & IUD- users (N=89) & $\begin{array}{c}\text { COC- users } \\
(\mathbf{N}=\mathbf{4 5})\end{array}$ & $\begin{array}{c}\text { Injectables users } \\
\mathbf{( N = 1 6 )}\end{array}$ & $\boldsymbol{p}$ value \\
\hline Mean \pm SD & $5.34 \pm 5.38$ & $5.51 \pm 5.46$ & $3.24 \pm 1.98$ & $3.03 \pm 1.64$ & 0.02 \\
\hline & & $p$ value is significant $<0.05$ & & \\
\hline
\end{tabular}

\section{Table 5. Seropositivity among users}

\begin{tabular}{|c|c|c|c|c|c|}
\hline & & $\begin{array}{c}\text { Non-users } \\
(\mathrm{N}=50)\end{array}$ & $\begin{array}{c}\text { IUD-users } \\
(\mathrm{N}=89)\end{array}$ & COC- users V & $\begin{array}{c}\text { Injectable users } \\
(\mathrm{N}=16)\end{array}$ \\
\hline \multirow{3}{*}{ Seropositive } & RF only & $7(14 \%)$ & $11(12.6 \%)$ & $11(24.4 \%)$ & $2(12.5 \%)$ \\
\hline & Both RF \& ACPA & $31(62 \%)$ & $43(48.3 \%)$ & $15(33.3 \%)$ & $2(12.5 \%)$ \\
\hline & ACPA only & $4(8 \%)$ & $24(27 \%)$ & $7(15.6 \%)$ & $8(50 \%)$ \\
\hline
\end{tabular}

\section{Table 6. Laboratory investigations among studied patients}

\begin{tabular}{|c|c|c|c|c|c|}
\hline $\begin{array}{c}\text { Laboratory } \\
\text { investigation } \\
\text { (Mean } \pm \text { SD) }\end{array}$ & Non-users $(\mathrm{N}=50)$ & IUD-users $(\mathrm{N}=89)$ & COC-users $(\mathrm{N}=45)$ & $\begin{array}{l}\text { Injectable users } \\
\qquad(\mathrm{N}=16)\end{array}$ & p value \\
\hline ESR & $38.46 \pm 25.896$ & $36.25 \pm 18.327$ & $41.64 \pm 17.971$ & $34.69 \pm 11.026$ & 0.453 \\
\hline CRP & $14.28 \pm 16.445$ & $9.020 \pm 8.946$ & $9.69 \pm 12.134$ & $10.42 \pm 8.713$ & 0.09 \\
\hline RF & $71.504 \pm 98.463$ & $63.29 \pm 72.405$ & $98.09 \pm 150.505$ & $34.19 \pm 55.680$ & 0.118 \\
\hline ACPA & $133.57 \pm 166.125$ & $201.26 \pm 179.973$ & $118.51 \pm 165.222$ & $124.86 \pm 117.968$ & 0.021 \\
\hline \multicolumn{6}{|c|}{$p$ value is significant $<0.05$} \\
\hline
\end{tabular}

Table 7. DAS28/CRP and its components, RASS and SES among studied patients

\begin{tabular}{|c|c|c|c|c|c|c|c|} 
Mean \pm SD & $\begin{array}{c}\text { Non-users } \\
\mathrm{N}=50\end{array}$ & $\begin{array}{c}\text { IUD-users } \\
\mathrm{N}=89\end{array}$ & $\begin{array}{c}\text { OC-users } \\
\mathrm{N}=45\end{array}$ & $\begin{array}{c}\text { Injectables- } \\
\text { users } \mathrm{N}=16\end{array}$ & $\begin{array}{c}\text { All patients } \\
\mathrm{N}=200\end{array}$ & p value \\
\hline DAS28/CRP & $3.63 \pm 1.87$ & $4.26 \pm 1.26$ & $4.03 \pm 1.47$ & $4.41 \pm 1.24$ & $4.06 \pm 1.49$ & 0.085 \\
\hline VAS (0-100) & $41.92 \pm 32.48$ & $53.04 \pm 23.98$ & $53.53 \pm 27.87$ & $55.00 \pm 18.86$ & $50.53 \pm 27.16$ & 0.079 \\
\hline RASS & $60.34 \pm 51.43$ & $83.73 \pm 44.92$ & $77.04 \pm 39.52$ & $96.88 \pm 28.68$ & $77.43 \pm 45.52$ & 0.008 \\
\hline SES & $9.54 \pm 7.75$ & $9.18 \pm 7.39$ & $8.33 \pm 5.96$ & $8.81 \pm 3.90$ & $9.05 \pm 6.94$ & 0.857 \\
\hline \multicolumn{7}{|c|}{$p$ value is significant $<0.05$} \\
\hline
\end{tabular}


statistically significant difference between the studied subgroups and so was the RA disease activity expressed by DAS28/CRP between the four subgroups of patients.

There is positive correlation between ACPA titers and RASS ( $\mathrm{r}=0.208, \mathrm{p}=0.003)$ Table 8 .

\section{Discussion}

Rheumatoid arthritis (RA) is a chronic inflammatory autoimmune disease. A highrisk genetic background, in combination with epigenetic marks and environmental exposures, leads to a cascade of that can induce synovitis with consequent destructive arthritis. A variety of extra-articular affection can also occur [5].

RA disease is more common in women than in men: female to male ratio is around 3:1 suggesting role for sex hormones in disease pathogenesis [16].

The issue of contraception and contraceptive method safety is of paramount importance in female patients with RA given the fact that the disease onset often occurs during the reproductive years. In addition, some treatments for RA are teratogenic, necessitating highly effective contraception for sexually active females [32]. Besides, many of the patients could have articular impairments that could interfere with raising greater number of offspring, thus the need for highly effective contraception among sexually active RA patients [53].

As we conducted the study, we showed the prevalence of use of each contraceptive method among the married RA patients who were on contraception for $>6$ months and were enrolled in our study; IUD (59.33\%), COC (30\%), injectables $(10.67 \%)$.

This came in agreement with results from Egypt Demographic and Health Survey (EDHS), which showed that the most widely used method is the intrauterine device (IUD), followed by the combined oral contraceptive (COC) pill then the progestin injectables; the prevalence of each contraceptive method among the contraceptive users as: IUD (50.84\%), COC $(27.11 \%)$ and injectables (15.25\%) [54].

The presence of citrullinated proteins, initially considered specific for the RA synovium, was later observed in non-RA inflammatory conditions; citrullination has been reported to be a process present in a wide range of inflammatory conditions, suggesting that this is an inflammation-dependent rather than diseasedependent process. This is in agreement with the concept of inflammation as common soil of the multifactorial diseases, encompassing several chronic inflammatory rheumatic disorders [55]. The ACPA is considered a predictive antibody to detect individuals at risk for RA and was found to be positive before the clinical onset of RA; It is also considered as a diagnostic, as well as a prognostic (predicting outcome/progression) biomarker; It is correlated with RA disease activity parameters, and has been proven that there is a strong correlation between greater disease activity and degree of radiographic damage and anti-CCP positivity [56].

In our study, it was found that the percentage of IUD-using patients who were ACPA seropositive was $75.3 \%$ which is the highest among all studied subgroups. Besides, the ACPA titer was significantly higher among the IUD-users compared to other subgroups in the study.

Also, $75.5 \%$ of all RA patients were exposed to prior use of IUD before the onset of RA. The ACPA titer was significantly higher among the subgroup of patients who used IUD someday prior to RA onset compared to those with disease onset preceding IUD use.

Furthermore, the disease duration in IUDusers -and even in other methods-users who used IUD earlier in their lives- was significantly longer than never IUD-users i.e. the earlier the first exposure to IUD, the longer the disease duration, suggesting that chronic inflammation that triggered citrullination and ACPA production will perpetuate and eventually present clinically.

Table 8. Correlations between RF/ACPA titers and disease activity and severity

\begin{tabular}{|c|c|c|c|c|}
\hline \multicolumn{2}{|l|}{ Parameter } & DAS & RASS & SES \\
\hline \multirow{2}{*}{ ACPA in all patients $(N=200)$} & $r$ & 0.133 & 0.208 & 0.097 \\
\hline & $\mathrm{p}$ & 0.061 & 0.003 & 0.172 \\
\hline \multirow{2}{*}{ RF in all patients $(\mathrm{N}=200)$} & $r$ & 0.073 & 0.026 & 0.032 \\
\hline & $\mathrm{p}$ & 0.304 & 0.719 & 0.655 \\
\hline \multicolumn{5}{|c|}{$p$ value is significant $<0.05$} \\
\hline
\end{tabular}


To our knowledge, there is no current studies examining the direct effect of IUD use on female RA patients, however, our findings came in consistence with the Studies of the Etiology of Rheumatoid Arthritis (SERA) that showed that first degree female relatives of RA (who were not RA patients) who were currently using an IUD had a statistically significantly increased risk for anti-CCP positivity. They also found a trend toward a decreased risk of anti-CCP positivity in women who used combined oral contraceptive (COC) pills, either currently or in the past. An association between current IUD use and a higher prevalence of anti-CCP positivity in the blood was suggested. However, this study was not able to address whether IUDs increase the risk of actually developing RA; they suggested that IUDs may be associated with the development of RA-related autoantibodies in some women who are at increased risk for the disease [43].

The mechanisms that link contraceptive factors to ACPA generation still need elucidation; IUDs have been shown to generate endometrial inflammatory responses, therefore, the association of IUD use and ACPA suggests that IUD-induced endometrial inflammation may be a potential mucosal trigger of RA-related antibodies in women at risk for RA. Moreover, recent data suggest that ACPA may be directly pathogenic, and thus it is important to understand factors that influence the development of these RA-related autoantibodies, even in the absence of inflammatory arthritis. These suggestions may make the IUD choice an unfavourable one in RA patients if it shows a future relation between RA activity and IUD use.

Doran and colleagues, in 2004 also observed an inverse association between ever-use of OC and the risk of RA. They also showed that the risk of developing RA is lower when OC exposure occurred in earlier years, which suggests that the higher doses of estrogens and progestins contained in earlier OC preparations may have a stronger protective effect against developing RA [57]; the most recent study of S, which included women aged 18 and above, living in a defined area of Sweden between 1996 and 2014 revealed that the ever and past COC use decreased the risk of RA, especially ACPA-positive RA. Besides, a long duration of $\mathrm{COC}$ use decreased the risk of both disease subsets, suggesting that combined hormonal contraceptive methods represent a safe and effective option for patients with RA [35].

In our study, ACPA titers were significantly higher among IUD users compared to COC users; the disease duration in IUD users was significantly higher than COC users; the COC users had the least RASS, however, we could not clearly show whether COC has a protective role in RA or not. Larger scale studies with emphasis on the past and current use of COC is needed to explore possible protective role for COC in RA patients.

RA disease activity was assessed using the Disease Activity Score-28 using C-reactive protein (DAS 28/CRP) and RA disease severity using the Rheumatoid Arthritis Severity Scale (RASS), which is a physician-completed scale consisting of three visual analogue scales (VAS): disease activity, functional impairment and physical damage. The previous parameters in addition to different clinical, laboratory and radiographic parameters were compared and correlated with differently used contraceptive methods among RA female patients.

There was significant difference in RASS between the four subgroups; it was highest in injectables users followed by the IUD users which was also significantly higher than non-users. Moreover, there is positive correlation between ACPA titre and RASS.

Initially, we need to report that the subgroup of patients who were using injectables was a strikingly unique group; they were the group that $75 \%$ of them were seronegative; however, they had the highest RASS, which is one of the severity parameters in our study; on the other hand, there was no significant difference between their SES scale and the other subgroups.

This result can raise some questions about their significance: injectables contraceptives contain DMPA which was found to be associated with osteopenia according to various studies $[58,59,44]$. RASS is a composite measure of three visual analogue scales (VAS) for disease activity, functional impairment and physical damage. The higher RASS that was encountered by injectable users can be explained by the proposed osteopenia caused by the DMPA which is the component of the injections might be an aggravating factor of pain; this may be translated by the physician as a more severe disease activity and functional impairment, as the examiner may address them as higher disease activity, that would result in more functional impairment and when plotting that on the scale, higher values would be assumed. But as we did not investigate bone density in all patients or the individual 
components of the RASS, we cannot generalize this observation.

Herein, we highlighted the importance of using an appropriate contraceptive method in RA female patients taking into consideration the possible impact of each contraceptive method as regards disease activity, severity and/or damage. However, gynecologic assistance to provide evidence for presence of chronic inflammation can be a limitation of the study which can be taken into consideration in future research.

\section{Conclusion}

The increased ACPA positivity and higher ACPA titers in women who are currently IUD users suggests a possible etiopathogenic role for IUD in onset and perpetuation of RA disease; mechanisms by which IUD could increase risk for RA-related autoimmunity need further study; cessation of IUD use in RA patients might improve their current state of disease.

Possible protective role for COC in female RA patients that require further elucidation by larger scale studies and emphasis on the past and current $\mathrm{COC}$ use and duration of use.

\section{Recommendations}

Given the importance of effective safe contraceptive use by RA patients, further future large scale population-based studies are needed to evaluate the effect of long-term contraceptive use and RA disease activity and severity. Larger numbers of newly diagnosed RA patients have to be enrolled to elucidate the correlation between various contraceptive methods and RA.

\section{References}

1. Bellucci E, Terenzi R, Paglia GM et al. One year in review 2016: pathogenesis of rheumatoid arthritis. Clin. Exp. Rheumatol. 34, 793-801 (2016).

2. McInnes IB, Schett G. Mechanisms of Disease: The Pathogenesis of Rheumatoid Arthritis. N. Engl. J. Med. 365, 2205-19 (2011).

3. Plenge RM. Genomic and Personalized Medicine. Rheumatoid. Arthritis. 2, 839-852.

4. Dekkers J, Toes RE, Huizinga TW et al. The role of anticitrullinated protein antibodies in the early stages of rheumatoid arthritis. Curr. Opin. Rheumatol. 28(3), 275-81 (2016).

5. Angelotti F, Parma A, Cafaro G et al. One year in review 2017: pathogenesis of rheumatoid arthritis. Clin. Exp. Rheumatol. 35(3), 368-378 (2017).

6. Gerstner C, Dubnovitsky A, Sandin C et al. Functional and structural characterization of a novel HLADRB1*04:01-restricted $\bigotimes$-enolase $\mathrm{T}$ cell epitope in rheumatoid arthritis. Front. Immunol. 7, 494 (2016).
7. Araki Y, Mimura T. The Mechanisms Underlying Chronic Inflammation in Rheumatoid Arthritis from the Perspective of the Epigenetic Landscape. J. Immunol. Res. 2016 (2016).

8. Joseph R, Jose Raj MG, Sundareswaran S et al. Does a biological link exist between periodontitis and rheumatoid arthritis? World. J. Rheumatology. 4(3), 8087 (2014).

9. Wu X, He B, Liu J et al. Molecular Insight into Gut Microbiota and Rheumatoid Arthritis. Int. J. Mol. Sci. 17(3), 431 (2016).

10. Wegner N, Lundberg K, Kinloch A et al. Autoimmunity to specific citrullinated proteins gives the first clues to the etiology of rheumatoid arthritis. Immunol. Rev. 233, 34-54 (2010).

11. Klareskog L, Malmstrom V, Lundberg $\mathrm{K}$ et al. Smoking, citrullination and genetic variability in the immunopathogenesis of rheumatoid arthritis. Semin. Immunol. 23, 92-98 (2011).

12. Catrina AI, Ytterberg AJ, Reynisdottir G et al. Lungs, joints and immunity against citrullinated proteins in rheumatoid arthritis. Nat. Rev. Rheumatol. 10, 645-653 (2014).

13. Koziel J, Mydel P, Potempa J. The link between periodontal disease and rheumatoid arthritis: an updated review. Curr. Rheumatol. Rep. 16, 408 (2014).

14. Valesini G, Gerardi MC, Iannuccelli C et al. Citrullination and autoimmunity. Autoimmun. Rev. 14(6), 490-497 (2015).

15. Hensvold AH, Reznisdottir G, Catrina A. From citrullination to specific immunity and disease in rheumatoid arthritis. Protein. Deimin. Human. Health. Dis. 2, 25-42 (2014).

16. Oliver JE, Silman AJ. Why are women predisposed to autoimmune rheumatic diseases? Arthritis Research \& Therapy. 11(5), 252 (2009).

17. Cutolo M, Villaggio B, Seriolo B et al. Synovial fluid estrogens in rheumatoid arthritis. Autoimmun. Rev. 3, 193-8 (2004).

18. Castagnetta LA, Carruba G, Granata OM et al. Increased estrogen formation and estrogen to androgen ratio in the synovial fluid of patients with rheumatoid arthritis. $J$. Rheumatol. 30, 2597-605 (2003).

19. Straub RH, Harle P, Sarzi-Puttini P et al. Tumor necrosis factor-neutralizing therapies improve altered hormone axes: an alternative mode of anti-inflammatory action. Arthritis. Rheum. 54, 2039-46 (2006).

20. Khalkhali-Ellis Z, Seftor EA, Nieva DR et al. Estrogen and progesterone regulation of human fibroblast-like synoviocyte function in vitro: implications in rheumatoid arthritis. J. Rheumatol. 27, 1622-31 (2000).

21. Schmidt M, Hartung R, Capellino S et al. Estrone/17Estradiol Conversion to, and Tumor Necrosis Factor Inhibition by, Estrogen Metabolites in Synovial Cells of Patients With Rheumatoid Arthritis and Patients With Osteoarthritis. Arthritis \& Rheumatism. 60 (10), 29132922 (2009).

22. Straub RH. The complex role of estrogens in inflammation. Endocr. Rev. 28, 521-74 (2007). 
23. Cutolo M, Seriolo B, Villaggio B et al. Androgens and estrogens modulate the immune and inflammatory responses in rheumatoid arthritis. Ann. N. Y. Acad. Sci. 966, 131-42 (2002).

24. Gigalis S, Hahn S, Hasler P. The NET outcome: are neutrophil extracellular traps of any relevance to the pathophysiology of autoimmune disorders in childhood? Front. Pediatr. 4, 97 (2016)

25. Corsiero E, Bombardieri M, Carlotti E et al. Single cell cloning and recombinant monoclonal antibodies generation from RA synovial B cells reveal frequent targeting of citrullinated histones of NETs. Ann. Rheum. Dis. 75, 1866-75 (2016).

26. Papadaki G, Kambas K, Choulaki C et al. Neutrophil extracellular traps exacerbate Th1-mediated autoimmune responses in rheumatoid arthritis by promoting DC maturation. Eur. J. Immunol. 46, 2542-54 (2016).

27. Sorice M, Iannuccelli C, Manganelli V et al. Autophagy generates citrullinated peptides in human synoviocytes: a possible trigger for anti-citrullinated peptide antibodies. Rheumatology (Oxford) 55, 1374-85 (2016).

28. van Loosdregt J, Rossetti M, Spreafico R et al. Increased autophagy in CD4+ $\mathrm{T}$ cells of rheumatoid arthritis patients results in Tcell hyperactivation and apoptosis resistance. Eur. J. Immunol. 46, 2862-70 (2016).

29. Kharlamova N, Jiang X, Sherina N et al. Antibodies to porphyromonas gingivalis indicate interaction between oral infection, smoking, and risk genes in rheumatoid arthritis etiology. Arthritis. Rheum. 68, 604-13 (2016).

30. Li S, Yu Y, Yue Y. Autoantibodies from single circulating plasmablasts react with citrullinated antigens and porphyromonas gingivalis in rheumatoid arthritis. Arthritis. Rheumatol. 68, 614-26 (2016).

31. Lu MC, Yu CL, YU HC et al. Anti-citrullinated protein antibodies promote apoptosis of mature human Saos-2 osteoblasts via cellsurface binding to citrullinated heat shock protein 60. Immunobiology. 221, 76-83 (2016).

32. Kahlenberg JM, Fox DA. Advances in the Medical Treatment of Rheumatoid Arthritis. Hand. Clinics. 27(1), 11-20 (2011).

33. Sammaritano LR, Bermas BL. Contraception and Pregnancy in Patients with Rheumatic Disease. Springer Science and Business Media (2014).

34. Jensen JT, Mishell DR. Family planning: contraception, sterilization, and pregnancy termination: Comprehensive Gynecology; 6th edition. Philadelphia, PA: Elsevier Mosby (2012).

35. Orellana C, Saevarsdottir S, Klareskog L et al. Oral contraceptives, breastfeeding and the risk of developing rheumatoid arthritis: results from the Swedish EIRA study. Ann. Rheum. Dis. 76(11), 1845-1852 (2017).

36. Ortiz ME, Croxatto HB. Copper-T intrauterine device and levonorgestrel intrauterine system: biological bases of their mechanism of action. Contraception. 75(6 Suppl), 16-30 (2007)

37. Lewis RA, Taylor D, Natavio MF et al. Effects of the levonorgestrel-releasing intrauterine system on cervical mucus quality and sperm penetrability. Contraception. 82(6), 491-6 (2010).
38. Ortiz ME, Croxatto HB, Bardin CW. Mechanisms of action of intrauterine devices. Obst. Gynecol. Surv. 51(12 Suppl), S42-51 (1996)

39. Martins GG, Junior JE, Tomaz T et al. The risk of genital infections in women using intrauterine device. DST - J Bras Doenças Sex Transm. 28(2), 61-63 (2016).

40. Madden T, Grentzer JM, Secura GM et al. Risk of Bacterial Vaginosis in Users of the Intrauterine Device: A Longitudinal Study. Sex. Transm. Dis. 39(3), 217-222 (2012).

41. Arenas-Osorio AE, Osorio-Arcila Y, Cardona-Arias J. Prevalence of vaginal Actinomycosis and its association with the use of the intrauterine device in three health institutions in Antioquia. CES Med. 29(1), 47-58 (2013).

42. Kim YJ, Youm J, Kim JH et al. Actinomyces-like organisms in cervical smears: the association with intrauterine device and pelvic inflammatory diseases. Obstet. Gynecol. Sci. 57(5), 393-396 (2014).

43. Khatter S, Parish MC, Feser ML et al. Contraceptive Factors Are Associated with Serum Antibodies to Citrullinated Protein Antigens in Women at Elevated Risk for Future Rheumatoid Arthritis. Arthritis \& Rheumatology. 66, S1276 (2014).

44. Petitti DB, Piaggio G, Mehta S et al. Steroid hormone contraception and bone mineral density: a cross-sectional study in an international population: The WHO Study of Hormonal Contraception and Bone Health. Obstet. Gynecol. 95, 736-744 (2000).

45. Aletaha D, Neogi T, Silman AJ et al. Rheumatoid arthritis classification criteria: an American College of Rheumatology/European League Against Rheumatism collaborative initiative. Arthritis. Rheum. 62, 2569-81 (2010).

46. van der Heijde DM, van't Hof MA, van Riel PL et al. Judging disease activity in clinical practice in rheumatoid arthritis: first step in the development of a disease activity score. Ann. Rheum. Dis. 49, 916-20 (1990).

47. Bardwell WA, Nicassio PM, Weisman MH. Rheumatoid Arthritis Severity Scale: a brief, physician-completed scale not confounded by patient self-report of psychological functioning. Rheumatology. 41(1), 38-45 (2002).

48. Tefferi A. Primary Hematology; Transfusion Totowa. NJ: Humana Press. 41, 472 (2001).

49. Westergren A. The technique of the red cell sedimentation reaction. Am. Rev. Tuberc. 14, 94 (1926).

50. Anderson SG, Bentzon MW, Houba V et al. International reference preparation of rheumatoid arthritis serum. Bull. World. Health. Organ. 42, 311-8 (1970).

51. Schellekens GA, Visser H, De Jong BA et al. The diagnostic properties of rheumatoid arthritis antibodies recognizing a cyclic citrullinated peptide. Arthritis. Rheum. 43, 155 (2000).

52. Wolfe F, van der Heijde DM, Larsen A. Assessing radiographic status of rheumatoid arthritis: introduction of a short erosion scale. J. Rheumatol. 27, 2090-9 (2000).

53. Farr SL, Folger SG, Paulen ME et al. Safety of contraceptive methods for women with rheumatoid arthritis: a systematic review. Contraception. 82, 64-71 (2010). 
54. El-Zanatys. Ministry of Health and Population (MOHP), and ICF International (2015). Egypt Demographic and Health Survey (EDHS). Egypt Health Issues Survey 2015: Ministry of Health and Population (MOHP) Cairo, Egypt.

55. Scrino R, Vasile M, Bartosiwicz I et al. Inflammation as "common soil" of the multifactorial disease. Autoimmun. Rev. 10, 369-74 (2011).

56. Puszczewicz M, Iwaszkiewicz C. Role of anti-citrullinated protein antibodies in diagnosis and prognosis of rheumatoid arthritis. Arch. Med. Sci. 7(2), 189-194 (2011).
57. Doran MF, Crowson CS, O'Fallon WM et al. The effect of oral contraceptives and estrogen replacement therapy on the risk of rheumatoid arthritis: a population based study. J. Rheumatol. 31, 207-13 (2004).

58. Walsh JS, Eastell R, Peel NF. Effects of Depot medroxyprogesterone acetate on bone density and bone metabolism before and after peak bone mass: a casecontrol study. J. Clin. Endocrinol. Metab. 93(4), 1317-23 (2008).

59. Berenson AB, Radecki CM, Grady JJ et al. A prospective, controlled study of the effects of hormonal contraception on bone mineral density. Obstet. Gynecol. 98, 576-582 (2001). 